\title{
FITORREGULADORES NO AUMENTO DA FRUTIFICAÇÃO EFETIVA E PARTENOCARPIA EM PERAS CV. GARBER ${ }^{1}$
}

\author{
JACQUELINE CARDOSO TAVARES ${ }^{1}$, JOSÉ CARLOS FACHINELLO² ${ }^{2}$ JOAO BAPTISTA DA SILVA ${ }^{3}$, FLAVIO \\ GILBERTO HERTER ${ }^{4}$
}

\begin{abstract}
RESUMO - O trabalho foi realizado em pereiras da cultivar Garber (Pyrus communis x Pyrus pyrifolia), com o objetivo de verificar o efeito de ácido giberélico $\left(\mathrm{AG}_{3}\right.$ ), ácido naftalenoacético (ANA), aminoetoxivinilglisina (AVG) e tidiazurom (TDZ) no aumento da frutificação e na indução e desenvolvimento de frutas partenocárpicas. Durante a plena floração, aplicaram-se sobre os cachos florais 13 diferentes tratamentos: (50; 300 e 500 mg.L $\left.{ }^{-1}\right)$, ANA (50; 100 e 200 mg.L $\left.\mathrm{L}^{-1}\right)$, TDZ (5; 15 e 25 mg.L $\left.\mathrm{L}^{-1}\right)$ e AVG (50; 100 e 200 mg.L $\left.\mathrm{L}^{-1}\right)$, sendo que, na testemunha, foi aplicada somente água. A polinização entomófila foi controlada em $50 \%$ dos ramos. As variáveis avaliadas foram o número total e médio de frutas fixadas, peso fresco, número de sementes, deformação e coloração das frutas. Dentre os tratamentos, o TDZ, na concentração de 15 mg. $\mathrm{L}^{-1}$, proporcionou o maior o número total de frutas, número médio de frutas fixadas, promovendo aumento no peso e coloração das mesmas. As deformações foram superiores nas peras tratadas com TDZ e naquelas com menor quantidade de sementes por fruta.
\end{abstract}

Termos para indexação: Pyrus, hormônios.

\section{GROWTH REGULATORS ON THE INCREASE OF THE FRUIT SET AND PARTHENOCARPY ON GARBER PEAR}

ABSTRACT - This study was conduced with trees of pears Garber (Pyrus communis x Pyrus pyrifolia). The aim of this work was to examine the effects of growth regulators substances as gibberellic acid $\left(\mathrm{GA}_{3}\right)$, naphthaleneacetic acid, aminoetoxivinilglisine (AVG) and tidiazuron (TDZ) to increase the fruit set and to induce the parthenocarpy. During the period of full blossom, the bunches was pulverized with 13 different treatments: $\mathrm{GA}_{3}(50,300,500$ mg.L $\left.\mathrm{L}^{-1}\right)$, ANA $\left(50,100,200 \mathrm{mg} . \mathrm{L}^{-1}\right)$, TDZ $\left(5,15,25 \mathrm{mg} . \mathrm{L}^{-1}\right), \operatorname{AVG}\left(50,100,200 \mathrm{mg} . \mathrm{L}^{-1}\right)$ and a control treatment with water. The insect pollination, was controled in $50 \%$ of branches. It was determined the total number of fruits established, the average of fruits established per bunch, the weight of fruits and number of seeds per fruit. Between all treatments, the plants pulverized with TDZ, at a concentration of $15 \mathrm{mg} . \mathrm{L}^{-1}$, showed the highest number of fruits established and the highest average of fruits established per bunch. It was observed an increasing in the weight per fruit and the most intense color on plants with TDZ treatment. Pears that received TDZ treatments and those that presented less seeds per fruit showed greater deformity.

Index terms: Pyrus sp., plant growth regulators.

A baixa frutificação é um dos maiores problemas relacionados com a produção de pêra, pois tem como principal causa as limitações que envolvem o processo de polinização. A ausência de fertilização do óvulo em flores de pereira resulta em um menor número de frutas fixadas por planta e, portanto, redução na produtividade do pomar. Quando a polinização é insuficiente, há formação de reduzido número de sementes, e para que o ovário se desenvolva, torna-se necessário que a fruta utilize fitormônios produzidos em outras partes da planta, já que existem poucas sementes para realizar a produção local destas substâncias (Salisbury \& Ross, 1992). Desta forma, torna-se interessante a aplicação de fitorreguladores que venham a substituir o efeito dos fitormônios produzidos pelas sementes, promovendo o incremento na produção.

Segundo Browning (1991), em cultivares de pereira com tendência natural à partenocarpia, a presença de óvulos não é essencial para induzir o desenvolvimento partenocárpico. Assim, não é necessário que todos os óvulos de uma flor sejam fertilizados para que haja fixação da fruta. Muitas vezes, é necessária somente uma semente para promover o desenvolvimento deste órgão vegetal e algumas frutas podem desenvolver-se sem sementes. No entanto, Camargo (1978) observa que estas são pequenas, deformadas e sujeitas a abscisão.

Algumas cultivares de pereira são capazes de desenvolver frutas partenocárpicas através do estímulo de fitorreguladores para superar deficiências devido à incompatibilidade, insuficiência de polinização ou condições adversas no período de floração.

A frutificação efetiva em pereiras pode ser aumentada com a aplicação de 2,4,5-TP (3 a 6 mg.L $\left.\mathrm{L}^{-1}\right)$, na plena floração, tendo em vista que esta auxina sintática reduz a abscisão das frutas durante o período de queda natural (Gil et al., 1973; Looney, 1998). Outras substâncias pertencentes ao grupo das auxinas também são utilizadas para melhorar a frutificação em diversas espécies, como é o caso do ácido naftalenoacético (ANA), nas concentrações de 100 e $200 \mathrm{mg.L} \mathrm{L}^{-1}$, aplicado em pereiras da cultivar Le Conte, e do 2,4-D, que, em baixas concentrações, atua na fixação de frutas cítricas (Singh \& Sharma, 1994; Fachinello et al., 1996). Já o TDZ, substância que tem efeito semelhante a uma citocinina, na concentração de 5 a $10 \mathrm{mg}$. $\mathrm{L}^{-1}$, aplicado na floração, proporcionou aumento na frutificação e no tamanho das frutas em macieira e pereira, resultado semelhante aconteceu com o AVG aplicado na floração da macieira que proporcionou aumento da frutificação efetiva (Petri \& Leite, 1999),

O experimento foi conduzido no pomar didático do Centro Agropecuário da Palma, pertencente à Universidade Federal de Pelotas, localizado no município de Capão do Leão, Estado do Rio Grande do Sul, durante o ano de 2000, em pereiras da cultivar Garber enxertadas sobre Pyrus caleriana, com o objetivo de verificar o efeito do $\mathrm{GA}_{3}$, ANA, TDZ e AVG no aumento da frutificação efetiva com os seguintes tratamentos: testemunha (água); $\mathrm{GA}_{3}\left(50 ; 300\right.$ e $\left.500 \mathrm{mg} . \mathrm{L}^{-1}\right)$, ANA (50; 100 e 200 mg.L $\left.L^{-1}\right)$, TDZ (5; 15 e 25 mg.L.$\left.^{-1}\right)$ e AVG (50; 100 e 200 mg.L $\left.\mathrm{L}^{-1}\right)$,

As aplicações foram feitas no dia 19-09-2000, no período de plena floração, ou seja, quando mais de $75 \%$ das flores estavam abertas. A pulverização foi realizada com jato dirigido aos órgãos florais, sendo a testemunha pulverizada com água. Para controlar a polinização por insetos, $50 \%$ dos ramos foram revestidos por um túnel de tule.

O delineamento experimental utilizado foi o de blocos completamente casualizados, constituído por quatro blocos ao acaso, com plantas em cada bloco, sendo que, em cada planta, foram isolados ramos para receberem os tratamentos.

Para a determinação do índice de deformação, atribuíram-se notas de 1 a 4, de acordo com a severidade da deformação: a) nota 1 para fruta normal; b) nota 2 para fruta levemente deformada; c) nota 3 para fruta deformada; d) nota 4 para fruta muito deformada.

$\mathrm{Na}$ determinação do índice de coloração das frutas, foram atribuídas notas de 1 a 4, conforme a intensidade de coloração na epiderme.

As variáveis foram submetidas à análise da variância, pelo teste $\mathrm{F}$,

1 (Trabalho 052/2002). Recebido: 15/03/2001. Aceito para publicação: 26/10/2002.

2 Eng. Agr ${ }^{\circ}$ Mestre em Agronomia na FAEM - UFPEL. Fone: 0xx532722109. jacqueline@ zaz.com.br

3 Prof. Titular da FAEM - UFPEL, Caixa Postal 354, CEP 96001.970 Pelotas, RS, Brasil. jfachi@ ufpel.tche.br

4 Prof. Titular aposentado do IFM - UFPEL. Fone: 0xx532232662. jbsilva@ufpel.tche.br

5 Pesquisador da Embrapa Clima Temperado, Pelotas, RS. Fone: 2758134. herter@embrapa.cpact.br 
sendo que, em caso de resultado significativo, procedeu-se à comparação das médias através do teste de Duncan.

Para a realização das análises estatísticas, utilizou-se o programa Sanest (Zonta \& Machado, 1984).

A Tabela 1 mostra que, dentre os fitorreguladores testados, o TDZ demonstrou ser mais eficiente no aumento da frutificação efetiva. Os melhores resultados foram obtidos na concentração de $15 \mathrm{mg} . \mathrm{L}^{-1}$, superando em 11,75 vezes o número total de frutas fixadas na testemunha.

TABELA 1 - Efeito do uso de fitorreguladores sobre o número total de frutas fixadas, número de sementes, deformação e coloração das frutas aos 75 dias depois da plena floração, em pereiras da cv. Garber (FAEM - UFPel, 2000).

\begin{tabular}{|c|c|c|c|c|}
\hline \multirow{3}{*}{ TRATAMENTOS } & \multicolumn{4}{|c|}{ " AVALIAÇÃo } \\
\hline & FRUTAS & $\mathbf{N}^{0} \mathbf{D E}$ & DEFORMAÇÃO & COLORAÇÃO \\
\hline & FIXADAS & SEMENTES & NAS FRUTAS & DAS FRUTAS \\
\hline Testemunha & $4,253 \mathbf{d}$ & $8,8,010 \mathbf{a}$ & $\begin{array}{ll}1,083 \mathbf{c} \\
\end{array}$ & $1,338 \mathbf{b c}$ \\
\hline $\mathrm{GA}_{3}\left(50 \mathrm{mg} \cdot \mathrm{L}^{-1}\right)$ & $4,482 \mathbf{c d}$ & $7,578 \mathbf{a b}$ & $1,125 \mathbf{c}$ & 1,352 bc \\
\hline $\mathrm{GA}_{3}\left(300 \mathrm{mg} \cdot \mathrm{L}^{-1}\right)$ & $8,130 \mathrm{~cd}$ & $7,217 \mathbf{a b}$ & $1,213 \mathbf{b c}$ & $1,506 \mathbf{b c}$ \\
\hline $\mathrm{GA}_{3}\left(500 \mathrm{mg} \cdot \mathrm{L}^{-1}\right)$ & $14,518 \mathbf{b c}$ & $5,415 \mathbf{b}$ & $1,291 \mathbf{b c}$ & $1,648 \mathbf{b}$ \\
\hline ANA $\left(50 \mathrm{mg} \cdot \mathrm{L}^{-1}\right)$ & 10,218 bcd & $8,001 \mathbf{a}$ & $1,083 \mathbf{c}$ & $1,125 \mathbf{c}$ \\
\hline $\operatorname{ANA}\left(100 \mathrm{mg} \cdot \mathrm{L}^{-1}\right)$ & $4,552 \mathbf{c d}$ & $6,934 \mathbf{a b}$ & $1,156 \mathbf{c}$ & $1,305 \mathbf{b c}$ \\
\hline ANA $\left(200 \mathrm{mg} \cdot \mathrm{L}^{-1}\right)$ & 7,010 cd & $5,995 \mathbf{a b}$ & $1,406 \mathbf{b c}$ & $1,648 \mathbf{b}$ \\
\hline $\operatorname{AVG}\left(50 \mathrm{mg} \cdot \mathrm{L}^{-1}\right)$ & $3,437 \mathbf{d}$ & $6,869 \mathbf{a b}$ & $1,125 \mathbf{c}$ & $1,386 \mathbf{b c}$ \\
\hline $\operatorname{AVG}\left(100 \mathrm{mg} \cdot \mathrm{L}^{-1}\right)$ & $3,095 \mathbf{d}$ & $7,504 \mathbf{a b}$ & $1,113 \mathbf{c}$ & $1,213 \mathbf{c}$ \\
\hline $\operatorname{AVG}\left(200 \mathrm{mg} \cdot \mathrm{L}^{-1}\right)$ & $2,538 d$ & $7,293 \mathbf{a b}$ & $1,213 \mathbf{b c}$ & $1,125 \mathbf{c}$ \\
\hline $\operatorname{TDZ}\left(5 \mathrm{mg} . \mathrm{L}^{-1}\right)$ & $21,897 \mathbf{b}$ & $5,506 \mathbf{b}$ & $1,542 \mathbf{b}$ & $1,372 \mathbf{b c}$ \\
\hline TDZ $\left(15 \mathrm{mg} \cdot \mathrm{L}^{-1}\right)$ & $49,999 \mathbf{a}$ & $6,040 \mathbf{a b}$ & $2,048 \mathbf{a}$ & $2,419 \mathbf{a}$ \\
\hline TDZ (25 mg. $\left.\mathrm{L}^{-1}\right)$ & $52,525 \mathbf{a}$ & $6,002 \mathbf{a b}$ & $2,151 \mathbf{a}$ & $2,426 \mathrm{a}$ \\
\hline
\end{tabular}

Médias seguidas das mesmas letras, nas colunas, não diferem entre si, pelo teste de Duncan, ao nível de 5\% de significância.

A alta precipitação pluviométrica registrada durante a plena floração reduziu a polinização por insetos. Deve-se considerar também que temperaturas inferiores a $10^{\circ} \mathrm{C}$ paralisam a atividade das abelhas, $\mathrm{e}$ abaixo de $15^{\circ} \mathrm{C}$ já diminuem consideravelmente a efetividade da polinização (Faoro, 1994). A temperatura média diária durante a floração manteve-se predominantemente acima de $10^{\circ} \mathrm{C}$, porém houve períodos com temperatura inferior a $15^{\circ} \mathrm{C}$. Desta forma, a manifestação de frio e principalmente a ocorrência de chuvas contribuíram para reduzir a frutificação efetiva.

Outro aspecto a ser considerado diz respeito à autoincompatibilidade, tendo em vista que, no local onde foi realizado o trabalho e nas suas proximidades, não existem outras cultivares de pereira que pudessem servir como polinizadoras. Assim, praticamente todo o pólen responsável pela fertilização foi proveniente da própria cultivar Garber. Embora não seja conhecido o nível de autocompatibilidade da cultivar em estudo, acredita-se que este fato possa ter contribuído para reduzir a fertilização e, conseqüentemente, a fixação das peras.

Conforme se aumentaram os dias após a aplicação dos produtos, diminuiu-se o número de frutas fixadas. Este decréscimo em todos os tratamentos já era esperado, pois, conforme relato de Camargo (1978), em pomáceas, é normal ocorrer a queda natural das flores não fecundadas ou com problemas de polinização e ausência de fertilização. Também caem frutos devido à competição nutricional, sendo que somente as melhores frutas permanecem fixadas.Em todas as avaliações, o tratamento com $15 \mathrm{mg} . \mathrm{L}^{-1}$ de TDZ proporcionou as médias mais elevadas de peso, e coloração das frutas, porém com o maior índice de frutos defor- mados em relação à testemunha. Ao contrário do TDZ, tanto o ANA como o GA foram mais eficientes quando utilizados na concentração mais baixa (50 mg.L $\left.\mathrm{L}^{-1}\right)$, exceto aos 75 dias, quando GA 3 (500 mg.L $\left.\mathrm{L}^{-1}\right)$ mostrou melhor resultado se comparado às demais concentrações deste mesmo fitorregulador.

A partenocarpia foi verificada somente nos ramos com proteção à polinização entomófila, o que demonstra a eficiência dos insetos neste processo. As flores que receberam tratamento com GA $3\left(500 \mathrm{mg} . \mathrm{L}^{-1}\right) \mathrm{e}$ TDZ (25 mg. $\left.\mathrm{L}^{-1}\right)$ apresentaram um índice de $20 \%$ de partenocarpia, já as plantas pulverizadas com ANA (200 mg.L $\left.\mathrm{L}^{-1}\right)$, AVG (50 mg.L $\left.\mathrm{L}^{-1}\right)$, TDZ (5 mg. $\left.\mathrm{L}^{-1}\right)$ e TDZ (15 mg. $\left.\mathrm{L}^{-1}\right)$ tiveram $10 \%$ das frutas desprovidas de sementes. Nos demais tratamentos, não ocorreu desenvolvimento partenocárpico, sendo que as frutas apresentaram de 2 a 10 sementes.

Torna-se importante destacar que o número de sementes por fruta e o tamanho também estão correlacionados, pois o aumento de um implicou o acréscimo do outro. Esta correlação demonstra que as peras com maior número de sementes apresentaram maior peso fresco aos 75 dias após a plena floração.

O TDZ, na concentração de 15 mg. $L^{-1}$, mostra-se mais eficaz no aumento do número total de frutas fixadas, do número médio de frutas fixadas, do peso e coloração das frutas, porém contribui para maior incidência de deformações nas peras.

\section{REFERÊNCIASBIBLIOGRÁFICAS}

BROWNING, G. Gibberellin-induced parthenocarpic development of pedicels of Pyrus communis L. cv. Conference. Journal of Horticultural Science, Londres, v.66, n.2, p.177-181, 1991.

CAMARGO, U.A. Comportamento de algumas cultivares e seleções de macieira (Malus pumila Mill.) quanto à polinização na região Sul do Rio Grande do Sul. 1978. 62f. Dissertação (Mestrado em Agronomia - Fruticultura de Clima Temperado) - Faculdade de Agronomia Eliseu Maciel, UFPel, Pelotas-RS, 1978.

FACHINELLO, J.C.; NACHTIGAL, J.C.; KERSTEN, E. Fruticultura: fundamentos e práticas. Pelotas: Editora da UFPel, 1996. 311p.

FAORO, I.D. Queda de flores da pereira: algumas possíveis causas. Agropecuária Catarinense, Florianópolis, v.7, n.3, p.41-43, 1994.

GIL, G.F.; MARTIN, G.C.; GRIGGS, W.H. Fruit-set and development in the pear: diffusible growth substances from seeded and seedless fruits. Journal of the American Society for Horticulturae Science, Virginia, EUA, v.98, n.1, p.51-53, 1973.

LOONEY, N.E. Plant bioregulators in fruit production: an overview and outlook. Journal of Korean Society for Horticultural Science, Taejon, Coreia, v.39, n.1,p.125-128, 1998.

PETRI, J.L.; LEITE, G.B. Biorreguladores de crescimento na produção de frutas de clima temperado. In: II ENCONTRO NACIONAL SOBRE FRUTICULTURA DE CLIMA TEMPERADO, 2, 1999, Fraiburgo. Anais... Fraiburgo, SC: Empresa de Pesquisa Agropecuária e Extensão Rural de Santa Catarina S. A. - EPAGRI, p.123-130, 1999.

SALISBURY, F.B.; ROSS, C.W. Fisiologia vegetal. México: Iberoamérica, 1992.759p.

SINGH, J; SHARMA, K.K. Effect of chemicals on fruit set in "Le Conte" interspecific hybrid of common pear (Pyrus communis) and sand pear (P. Pyrifolia). Indian Journal of Agricultural Sciences, Nova Deli, v.64, n.10, p.711-712, 1994.

VEGO, D. The fruit-shape of pear in ralation to the seed contents. In: VIII INTERNATIONAL SYMPOSIUM ON PEAR, 8, 2000, Bologna. Abstracts... Bologna, Itália: International Society for Horticultural Science, p.265, 2000.

ZONTA, E.P.; MACHADO, A.A. Sanest: sistema de análise para microcomputadores. Registrado na secretaria especial de informática -SEI- sob n ${ }^{\circ}$ 066-060. Categoria A. Pelotas, RS, 1984. 